\title{
Contextual effects in motor adaptation adhere to associative learning rules
}

Guy Avraham¹,2, Jordan A. Taylor ${ }^{3}$, Assaf Breska ${ }^{1,2}$, Richard B. Ivry ${ }^{1,2}$, Samuel D. McDougle ${ }^{4}$

1. Department of Psychology, University of California, Berkeley, Berkeley, CA 94720, USA

2. Helen Wills Neuroscience Institute, University of California, Berkeley, Berkeley, CA 94720, USA

3. Department of Psychology, Princeton University, Princeton, NJ 08544, USA

4. Department of Psychology, Yale University, New Haven, CT 06520, USA

* Corresponding author

E-mail: guyavraham@berkeley.edu

Keywords: Sensorimotor adaptation; associative learning; cerebellum; forward model; RescorlaWagner model 


\section{ABSTRACT}

2 Two influential paradigms, eyeblink conditioning and motor adaptation, have deepened our

3 understanding of the theoretical and neural foundations of sensorimotor learning. Although both

4 forms of error-based learning are dependent on the cerebellum, the two literatures typically

5 operate within distinct theoretical frameworks. For eyeblink conditioning, the focus is on the

6 formation of associations between an error signal and arbitrary stimuli. For adaptation, the error

7 signal is used to modulate an internal model of a sensorimotor map for motor planning. Here we

8 take a step towards an integrative account of these two forms of learning, examining the relevance

9 of core concepts from associative learning for motor adaptation. Using a task that drives implicit

10 adaptation of reaching movements, we paired movement-related feedback with neutral auditory

11 or visual cues that served as conditioning stimuli (CSs). Trial-by trial changes in feedforward

12 movement kinematics exhibited two key signatures of associative learning: Differential

13 conditioning and compound conditioning. Moreover, after compound conditioning, a robust

14 negative correlation was observed between responses to the two elemental CSs of the compound

15 (i.e., overshadowing), consistent with the additivity principle posited by models of associative

16 learning. Computational modeling demonstrated that these results could not be captured by

17 conventional, context-insensitive algorithms used to describe motor adaptation. Associative

18 learning effects in motor adaptation provide a proof-of-concept for linking cerebellar-dependent

19 learning paradigms within a common theoretical framework.

\section{SIGNIFICANCE STATEMENT}

22 Motor adaptation is a cerebellar-dependent process, describing how the motor system remains

23 calibrated in response to environmental and bodily changes. Another cerebellar-dependent

24 learning phenomenon, eyeblink conditioning, is viewed as an associative learning process. Here

25 we sought to bringing together these two approaches for studying sensorimotor learning. We 
demonstrate that core associative learning phenomena are manifest during motor adaptation,

27 pointing to a common framework for these distinct cerebellar-dependent motor learning 28 processes.

\section{INTRODUCTION}

31 A hallmark of many motor learning tasks is the use of error information to improve performance.

32 Sensorimotor errors not only signal that an action has not been properly executed but can provide information on how the output of the system should be changed to increase the likelihood of future success in a similar context. Two paradigmatic tasks for studying error-based learning are sensorimotor adaptation and eyeblink conditioning. Sensorimotor adaptation refers to the gradual adjustment of movements in response to changes in the environment or body. The key principle in this process is that on each trial, sensorimotor prediction errors are used to update an internal model of a sensorimotor mapping to help keep the sensorimotor system precisely calibrated (Shadmehr and Krakauer, 2008; Wolpert et al., 1995; Wolpert and Ghahramani, 2000). In eyeblink conditioning, a form of classical conditioning (Pavlov, 1927), learning centers on the formation of associations between an aversive stimulus (i.e., an error signal) and arbitrary cues. In a standard variant of this task, an aversive air puff to the cornea (unconditioned stimulus, or US) elicits a

43 reflexive blink (unconditioned response, or UR). The US can be repeatedly paired with a predictive

44 conditioning stimulus (CS, such as a tone or light flash) to gradually induce a preemptive 45 conditioned eyeblink response (CR). The CR is adaptative, mitigating the anticipated aversive sensory consequences of the US. 
50 involves active planning (motor adaptation) and the other passive conditioning of a reflex (eyeblink

51 conditioning), both involve a learning signal based on a sensorimotor error that must occur close

52 in time to an initial prediction to drive learning (Brudner et al., 2016; Kitazawa et al., 1995;

53 Rasmussen et al., 2008; Schneiderman and Gormezano, 1964; Schween and Hegele, 2017;

54 Shadmehr et al., 2010). Second, both are strongly associated with the cerebellum (Donchin et al.,

55 2011; Garcia et al., 1999; Gerwig et al., 2007; Izawa et al., 2012; Kim and Thompson, 1997; Popa

56 et al., 2016; Schlerf et al., 2012).

While these parallels suggest a link between sensorimotor adaptation and eyeblink conditioning, it is surprising that the two literatures have generally operated within distinct theoretical frameworks. For adaptation, the focus has been on how an error signal is used to directly update a motor command and the anticipated sensory consequences of that command.

61 For eyeblink conditioning, the focus has been on how an error signal is used to build an association between an arbitrary stimulus (the CS) and an aversive event (the US) to produce a predictive, adaptive response (the $\mathrm{CR}$ ). This last point highlights one aspect of apparent divergence between adaptation and conditioning: A prominent feature of sensorimotor adaptation has been the finding that, at least when putatively restricted to implicit learning, arbitrary cues are not effective for this form of learning. For example, people do not differentially adapt their reaching movements to two opposing perturbations when the perturbation is signaled by a distinct color cue in the environment (Howard et al., 2012, 2013; Gandolfo et al., 1996). 
75 predictive, arbitrary sensory cues need not have a direct relationship to the events they predict,

76 accruing associative value as long as they comply with strict timing requirements (Schneiderman

77 and Gormezano, 1964; Smith et al., 1969).

Here we take a first step toward bridging these two worlds by applying some key

79 theoretical concepts and design features of classical conditioning studies to a visuomotor rotation

80 task. First, we test the idea that arbitrary sensory cues can indeed shape motor adaptation, even

81 though they have no direct link to motor commands. Second, we test the hypothesis that the effect

82 of these cues on adaptation will adhere to established principles of associative learning. By

83 demonstrating these parallels between conditioning and motor adaptation, we offer a more

84 parsimonious framework for understanding these disparate cerebellar-dependent learning 85 processes.

RESULTS

88 We modified a visuomotor rotation task to ask if implicit motor adaptation exhibits associative

89 behavior when a perturbation is paired with an arbitrary sensory cue. Participants were asked to

90 reach from a start location to a target with movement feedback provided by a cursor (Fig. 1A).

91 Drawing on classic work in animal learning (Pavlov, 1927; Rescorla and Wagner, 1972), we

92 presented sensory events (a tone or light stimulus) as imperative cues for movement initiation.

93 The use of a neutral stimulus to cue movement initiation provides a means to establish a tight

94 temporal link between these arbitrary cues - the CSs in the task - and sensorimotor feedback,

95 the US. This temporal linkage of the CS and US, where the CS onset precedes the US and the

96 two events occur close in time, are prerequisites for cerebellar-dependent delay conditioning

97 (Schneiderman and Gormezano, 1964). To promote this timing constraint, a warning was given if

98 the participant did not move within $400 \mathrm{~ms}$ of the imperative, reminding them to initiate the 
99 movement faster. Participants complies with this requirement, exhibiting rapid reaction times

100 (Mean \pm STD: $287 \pm 45.2 \mathrm{~ms}$ ).

101 Rather than impose a typical visuomotor perturbation on the feedback (e.g., rotate the 102 cursor relative to the true hand position), we opted to use "clamped" feedback. In this technique, 103 which is designed to isolate implicit motor adaptation, the cursor path is not tied to the direction 104 of the reach but rather follows an invariant ("clamped") path, with the radial position of the cursor 105 corresponding to the radial position of the hand (Morehead et al., 2017; Shmuelof et al., 2012). 106 As in past studies with clamped feedback, the participant was fully informed of the clamp 107 manipulation and instructed to ignore the task-irrelevant cursor and reach straight to the target on 108 all trials. As such, this method eliminates the potentially confounding effects of strategic processes 109 (McDougle et al., 2016; Kim et al., 2020). Nonetheless, participants' reach angles gradually shift 110 in the direction opposite to the clamp and shows the cardinal signatures of implicit motor 111 adaptation without awareness (Morehead et al., 2017; Kim et al., 2018, 2019; Parvin et al., 2018;

112 Tsay et al., 2020a, 2020b, 2021a, 2021b; Avraham et al., 2021; Poh et al., 2021; Vandevoorde 113 and Orban de Xivry, 2019; Yin and Wei, 2020). 
A

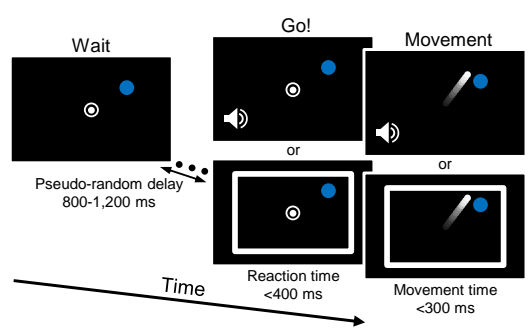

B

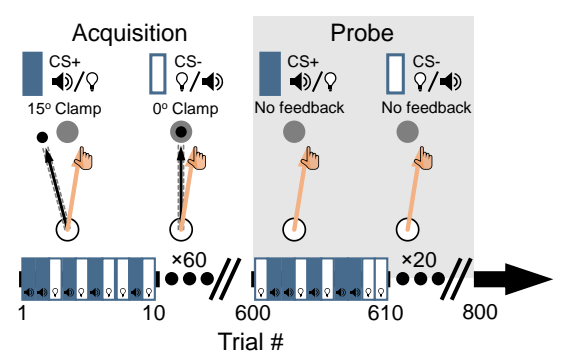

C

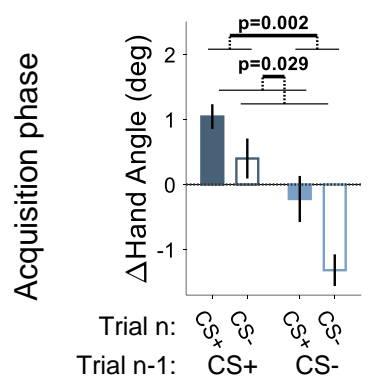

D

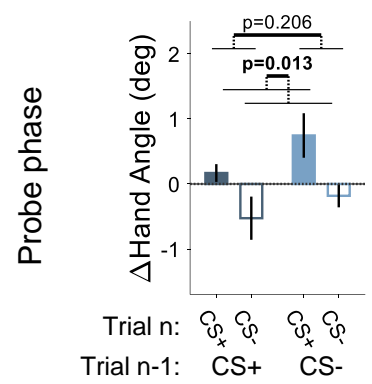

Pavlovian effect CS+- CS-

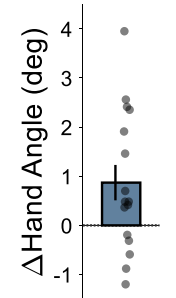

Pavlovian effect CS+- CS-

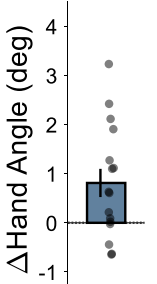

E Rescorla-Wagner

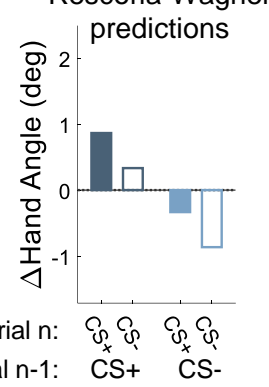

F Rescorla-Wagner

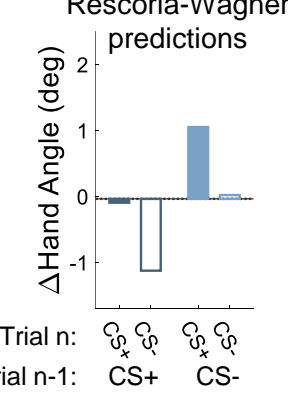

Figure 1. Experiment 1: Differential Conditioning.

(A) Reaching task. Participants reach from a start location (white circle) to a target (blue dot). Online feedback is provided by a cursor (white dot). The target is displayed in a fixed location for the entire experiment (location varied across participants), and the direction of the cursor trajectory is fixed ("clamped") on all trials. After a random delay, a tone or a light (white frame) is presented, serving as the movement imperative. The cue persists for the duration of the movement. (B) Experimental protocol. During acquisition (white background), a $15^{\circ}$ clamp (CW/CCW, counterbalanced across participants) was associated with CS+ (e.g., a tone) and a $0^{\circ}$ clamp with CS- (e.g., a light; counterbalancing the associations with the tone and light across participants). During the probe phase (gray background), CS+ and CS- were presented with no feedback. Throughout the entire experiment, CS+ and CS- trials were randomly interleaved. (C, D) Experimental results $(N=16)$ for trial-by-trial change $(\Delta)$ in hand angle during the acquisition $(C)$ and probe $(D)$ phases. Left panels present analysis results for an adaptation effect (main effect of trial $n-1$, dark vs light blue) and a Pavlovian effect (main effect of the presented CS on the current trial $n$, filled vs empty bars). The black outlined bar (right panel) presents the Pavlovian effect, i.e., the subtraction of hand angle changes between CS+ and CS- trials. (E, F) Rescorla-Wagner model simulation results during the acquisition $(E)$ and probe $(F)$ phases are consistent with the experimental results. Error bars represent SEM. Dots represent individual participants. 
135 In differential conditioning, one CS is paired with the US (CS+) and another CS is presented 136 without the US (CS-). Thus, only the CS+ should become associated with the US and result in a 137 CR. To implement this in Experiment 1, we used two arbitrary cues for the CS's - a tone or a light 138 cue - and clamped cursor feedback for the US's (Morehead et al., 2017). Rather than vary the presence or absence of feedback, we manipulated the trajectory of the cursor feedback to signal the presence or absence of an error: For the CS+ condition, the feedback cursor followed a clamped path that was rotated from the target by $15^{\circ}$ (i.e., error/US present); for the CS- condition, the feedback cursor always moved directly to the target (i.e., error/US absent). The CR in this scenario is the angular deviation of the movement in the opposite direction of the $15^{\circ}$ clamp.

During a 600-trial acquisition phase, CS+ and CS- trials were randomly interleaved. asymptote of $\sim 15^{\circ}$ (Fig. S1A). This rapid adaptation is consistent with previous adaptation studies,

147 particularly those in which the target appears at a fixed location (Bond and Taylor, 2015; Day et 148 al., 2016; McDougle et al., 2015, 2017; Poh et al., 2021).

The main analysis centered on trial-by-trial changes in hand angle. The change in hand angle from trial $n-1$ to trial $n$ is normally dictated by the feedback experienced in trial $n-1$. Thus, following experience with an error on CS+ trials, participants should show increased adaptation

152 (a positive change in hand angle), and following no error on CS- trials, decreased adaptation. We refer to these trial-by-trial changes as the "adaptation effect", the standard measure of learning in

154 sensorimotor adaptation studies. However, the conditioning framework makes a critical additional 155 prediction: The CS+ and CS- should differentially modulate the hand angle on trial $n$ itself. That 156 is, the presentation of the light or tone should produce a CR associated with that cue, leading to 157 a difference in hand angle between the two cues that is independent of the feedback during the 
previous trial. We refer to this as the "Pavlovian effect." Importantly, if implicit adaptation is not sensitive to arbitrary contextual cues, we expect to only observe an adaptation effect.

A robust adaptation effect was observed (Fig. 1C, left panel): Trial-by-trial changes in reaching direction ( $\Delta$ hand angle) were significantly affected by the CS presented on the previous trial $\left[F(1,15)=13.3, p=0.002, \eta_{p}^{2}=0.47\right]$ such that the change in hand angle was larger after $C S+$ trials compared to after CS- trials. That is, the error occurring on CS+ trials (US+) resulted in learning that carried over to the next trial, whereas the absence of an error on a previous CS- trial (US-) resulted in a relative reversion to baseline (extinction). This is the canonical signature of incremental motor adaptation.

The critical test of our hypothesis centers on the Pavlovian effect. As shown in Figure 1C (both panels), the results revealed clear Pavlovian effects: The hand angle increased in the direction of adaptation on CS+ trials (i.e., a positive change in hand angle relative to previous trials) and decreased on CS- trials $\left[F(1,15)=5.83, p=0.029, \eta_{p}^{2}=0.28\right]$. This effect provides a novel demonstration that arbitrary sensory cues can lawfully influence implicit motor adaptation.

We also observed an interaction between CS identity on trials $n-1$ and $n[F(1,15)=7.11$, $\left.173 \mathrm{p}=0.018, \mathrm{n}_{\mathrm{p}}^{2}=0.32\right]$. That is, the difference between $\mathrm{CS}+$ and CS- was larger on trials following a 174 CS- $\left(\right.$ Mean $\left.\pm S E, 1.09^{\circ} \pm 0.39^{\circ}\right)$ compared to trials following a CS $+\left(0.65^{\circ} \pm 0.35^{\circ}\right)$. This interaction 175 effect likely reflects an asymmetry between the rate of the acquisition and extinction processes 176 once CS-US associations are established. That is, the state following a CS+ trial may be closer 177 to its asymptotic limit than after a CS- trial and is thus more limited in its potential for further 178 change.

We note that the visual feedback was different on CS+ and CS- trials, with the cursor 180 deviating from the target in the former and moving in a straight line to the target in the latter. This 181 raises the possibility that the hand angle differences on CS+ and CS- trials could be affected by 
182 rapid online feedback responses. This explanation is unlikely given that the movements were 183 quite rapid ([Mean \pm STD], $103 \pm 31.2 \mathrm{~ms}$ ). To directly assess the online correction hypothesis, we 184 calculated the difference in hand angle $50 \mathrm{~ms}$ after movement initiation and at the radial distance 185 of the target. There was no overall change in hand angle between the time points $\left(-0.32^{\circ} \pm 2.92^{\circ}\right)$, 186 and no significant difference between CS+ and CS- trials on this metric $[t(15)=-0.47, p=0.640, d=-$ 187 0.12]. Thus, our results appear to pertain exclusively to feedforward learning. CS- trials were randomly presented in the absence of any visual feedback (no US). This phase provides a "clean" test for associative learning effects since it removes trial-by-trial effects that arise from the differential feedback given during CS+ and CS- acquisition trials. Here too we observed a significant Pavlovian effect: Although there was an overall decrease in hand angle across the probe phase (i.e., a partial washout of adaptation, Fig. S1A), there was a significant main effect of the CS presented on trial $n\left[F(1,15)=7.98, p=0.013, \eta_{p}{ }^{2}=0.35\right]$ (Fig. 1D), with a relative increase in hand angle on CS+ trials and a decrease on CS- trials. Moreover, there was neither an effect of the trial $n-1 \operatorname{CS}\left[F(1,15)=1.74, \mathrm{p}=0.206, \mathrm{n}_{\mathrm{p}}^{2}=0.10\right]$ nor a trial $n-1 \times n$ interaction $\left[F(1,15)=0.25, p=0.621, \eta_{p}^{2}=0.02\right]$, consistent with trial-by-trial adaptation being dependent on error feedback.

Importantly, the Pavlovian effect did not appear to be driven by explicit awareness of the

200 CS-US contingency. A post-experiment survey was used to classify participants as either aware 201 or unaware of the CS-US associations (see Methods). Participants who reported being aware of 202 the contingencies $(\mathrm{N}=7)$ did not show a different Pavlovian effect compared to those who reported 203 being unaware $(\mathrm{N}=9)$ in either the acquisition $\left(\mathrm{t}(14)=-0.333, \mathrm{p}=0.744, \mathrm{BF}_{10}=0.446, d=-0.17\right)$ or 204 probe $\left(\mathrm{t}(14)=-0.081, \mathrm{p}=0.937, \mathrm{BF}_{10}=0.431, d=-0.04\right)$ phases. A similar null effect of awareness on 205 the strength of Pavlovian conditioning has also been reported in studies of human delay eyeblink 206 conditioning (Clark and Squire, 1998). 
In summary, the observed effects of context on implicit motor adaptation in both the acquisition and probe phases in Experiment 1 are consistent with differential conditioning effects observed in classical conditioning. Feedforward implicit motor adaptation - here operationalized as a type of CR - was differentially modulated by an arbitrary sensory CS+ versus CS-, with a

211 greater response to the CS+, the cue that was paired with a visuomotor error.

The standard approach to describe motor adaptation is based on a "state-space" model 213 in which the motor state is updated according to the error observed on the previous trial. The 214 parameters of this model determine both the trial-by-trial retention of the previous state and the 215 rate of learning from the error (see Eq. 3 in the Methods). By definition, a model of this form only 216 predicts a behavioral change based on trial $n-1$ (i.e., an adaptation effect), where no change in 217 hand angle is produced by the trial $n \mathrm{CS}$; thus, this standard model cannot capture any context 218 effects (e.g., our Pavlovian effect). a straightforward account of context effects. It formalizes changes in conditioned responses via 221 the modulation of (arbitrary) learned associations. Here, the associative strengths, $V$, of the 222 conditioning stimuli are updated according to the learning rule (Eq. 1):

$$
V^{[n]}=V^{[n-1]}+\alpha \cdot \beta \cdot S P E^{[n-1]},
$$

224 where $V$ represents the associative strengths between the US and the CS. It is updated based on 225 the sensory prediction error (SPE) presented on trial $n-1$. The $S P E$ is defined as $\lambda-V^{n-1]}$, where $\lambda$ 226 is the maximum conditioning (asymptotic) level of the US. $\beta$ is the learning rate parameter of the 227 US and $\alpha$ represents the salience of the CS. We note that the Rescorla-Wagner model does not 228 provide a mechanistic account for error-correction itself (e.g., the fact that the motor system 229 "knows" to update movements in the direction opposite of the error). For simplicity, we assume 230 that the sign of the change in movement direction is determined by a specialized neural circuit 
231 attempting to reduce directional motor errors (Hadjiosif et al., 2021; Herzfeld et al., 2018; Wolpert 232 et al., 1998).

To illustrate that the Rescorla-Wagner model can capture differential conditioning behavior

234 similar to the results observed in Experiment 1, we performed model simulations. The simulation

235 results demonstrate both the adaptation effect and Pavlovian effect for the acquisition phase (Fig.

236 1E), as well as a clear Pavlovian effect for the Probe phase (Fig. 1F). Importantly, the behavioral

237 signature of differential conditioning, the larger changes in hand angle on CS+ trials compared to

238 CS- trials, holds for essentially all combinations of parameters in the Rescorla-Wagner model

239 (Fig. S2, left side). In contrast, there are no parameter combinations for the state-space model

240 that can produce this behavior (Fig. S2, right side). As noted above, the failure of the state-space

241 model should be expected given that it has no mechanism for handling contextual effects (we

242 return to this point in the Discussion).

To formally compare the Rescorla-Wagner and state-space models in terms of the

244 observed results, we conducted a model comparison by fitting each participant's data with the

245 two models. The Rescorla-Wagner model provided a better fit to the data than a standard state-

246 space model (Fig. S3; $t$-test comparing sum of squared residuals, $t(15)=-3.62, p=0.003, d=-0.90$;

$247 t$-test comparing Akaike Information Criterion (AIC) values, $t(15)=-6.44, p<0.001, d=-1.61)$.

An additional analysis provided further support for an associative learning account of the

251 the strength of these associations is constrained by the relevance of the cues; for instance,

252 gustatory cues are much more likely to be associated with an internal state (e.g., nausea) than a

253 visual cue (Garcia and Koelling, 1966). In the current study, the clamped feedback (the US) is a

254 highly relevant stimulus for reaching; as such, we should expect it to have an immediate strong 255 influence on motor behavior. In contrast, the imperative cues, the tone and light (the CSs) have 
256 no natural relevance for reaching; as such, their contribution to the CR should initially be quite

257 modest, gradually increasing over time (Fig. 2A). To test this prediction, we examined the time-

258 course of the adaptation and Pavlovian effects during acquisition using a linear mixed model

259 analysis. As expected, at early stages, the adaptation effect emerged quickly whereas the

260 contribution of the Pavlovian effect was small. Over experience, the relative contribution of the

261 two effects reversed (Type and Bin interaction effect: $F(1,365)=16.7, p<0.001$ ): The Pavlovian

262 effect gradually grew (mean slope, [95\% Cl]; 0.14, [0.04 0.24]) at the expense of the adaptation

263 effect, which eventually exhibited a reduced contribution (-0.15, [-0.25 -0.05]) (Fig. 2B). We note

264 that both the decrease in the adaptation effect and the increase in the Pavlovian effect are not

265 captured by a typical state-space model (Fig. S4).
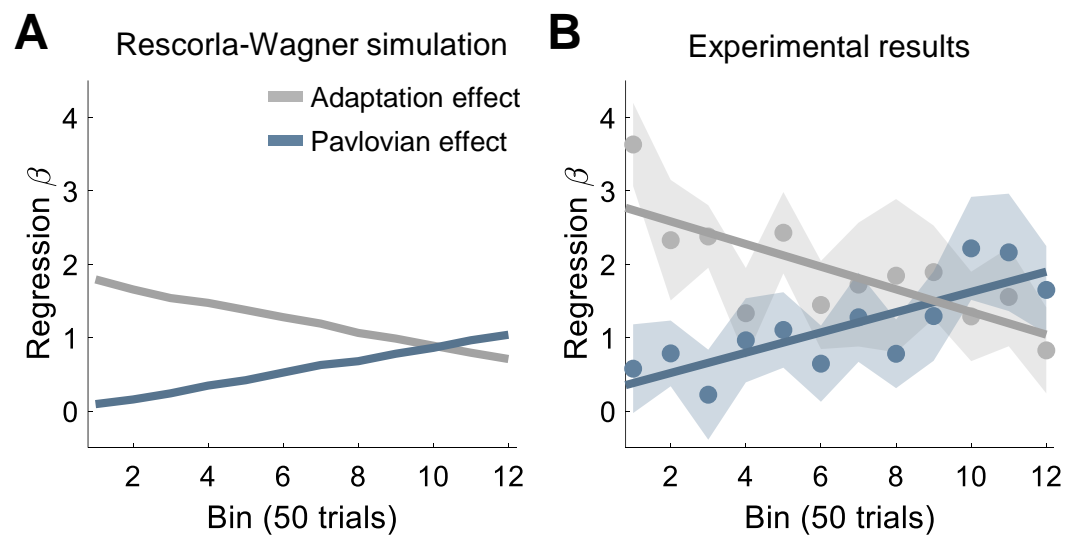

268 Figure 2. Dynamics of adaptation and Pavlovian effects.

269 Time course of the mean weights (regression $\beta$ ) of the adaptation (trial $n-1$, grey) and Pavlovian (trial $n$, 270 blue) effects in Experiment 1 as predicted by the Rescorla-Wagner model (A) and derived from fits of the 271 experimental results (B). Solid lines in B represent least squares regression lines. Shaded region represent 272 SEM. 
274 Additivity principle in response to compound stimuli is observed in motor adaptation

275 The differential conditioning results of Experiment 1 show that implicit motor adaptation

276 manifests a prominent feature of classical conditioning, the gradual associability of an error signal

277 with arbitrary sensory cues. In Experiment 2, we tested a second core phenomenon of associative

278 learning, the principle of additivity (Mackintosh, 1976; Pavlov, 1927; Rescorla and Wagner, 1972).

279 This principle is based on the idea that there is an associative capacity for a given US - the $V$

280 term in the Rescorla-Wagner equation. Multiple CSs can become associated with a given US, but

281 the combined associative strength is bounded by $V$. As a result of this capacity constraint, CSs

282 effectively compete with one another, with the associative strength split among multiple cues.

The classic method to test for additivity is compound conditioning, where two or more

284 stimuli are presented simultaneously to form a "compound" CS (Eq. 2). When paired with a US,

285 this compound CS will come to elicit CRs. Importantly, the associative strength of the Compound

$286 \mathrm{CS}\left(V_{\text {comp }}\right)$ is the sum of the associative strengths of the elemental CSs $\left(V_{i}\right)$, where $n S$ in Eq. 2

287 represents the number of elements forming the Compound CS. Consequently, each element of

288 the compound, when presented alone, elicits a proportionally weaker CR, with the degree of 289 attenuation being a function of the associative strength of that CS.

(2) $V_{i}^{[n]}=V_{i}^{[n-1]}+\alpha_{i} \cdot \beta \cdot\left(\lambda-V_{\text {comp }}^{[n-1]}\right) ; V_{\text {comp }}^{[n-1]}=\sum_{i=1}^{n S} V_{i}^{[n-1]}$

The additivity principle has received ample support in behavioral and neural studies of associative learning (Giurfa, 2007; Kehoe et al., 1994; Kehoe and Schreurs, 1986; Rescorla and Wagner, 1972; Weiss, 1972), but has not, to our knowledge, been tested in motor adaptation. In

294 Experiment 2 we used a compound conditioning design pairing a $15^{\circ}$ error clamp stimulus with a 295 compound CS (simultaneous presentation of the tone and light; Fig. 3A) on all trials during the 296 acquisition phase. As in Experiment 1, we again observed robust adaptation in the acquisition 297 phase, manifest as a change in hand angle in the direction opposite to the clamp (Fig. S1). 
A

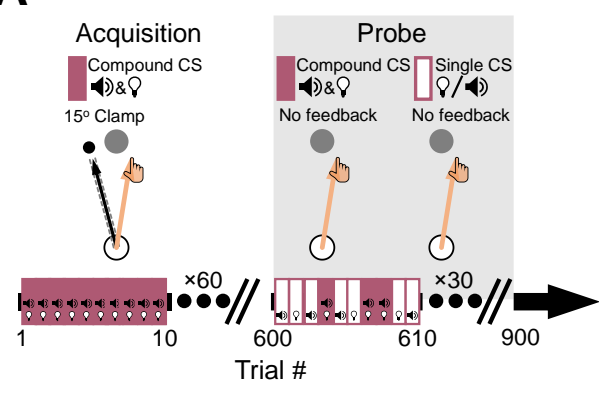

B

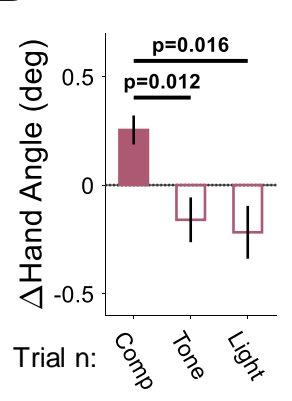

C Rescorla-Wagner

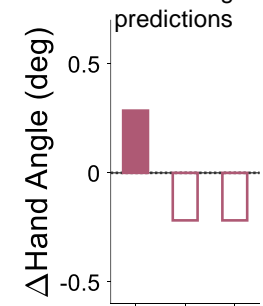

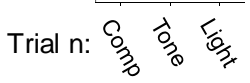

D

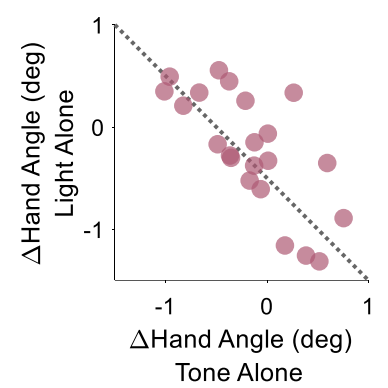

E

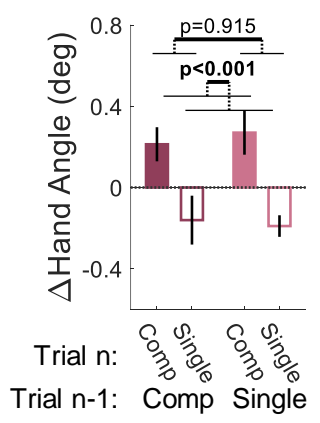

Figure 3. Experiment 2: Compound Conditioning.

(A) During acquisition, a tone and light were presented simultaneously (Compound CS), serving as the imperative signal for the reaching movement. They were paired on all trials with a $15^{\circ}$ clamp. During the probe phase, no feedback was provided, and the CSs were presented either together (Compound CS) or alone (Single CS; tone or light). (B) Experimental results $(\mathrm{N}=22)$ for the $\Delta$ hand angle during the probe phase, showing a positive relative change for the compound CS on the current trial $n$ (filled bar) and a negative relative change for each of its elements (empty bars). (C) Predictions from the Rescorla-Wagner model for trial-by-trial $\Delta$ hand angle during the probe phase. Note that the two elements were assumed to have equal weight in the simulation. (D) Scatter plot showing the between-participant trade-off in terms of the associative strength of the two CSs (the dotted black line represents the unity line). (E) The $\Delta$ hand angle after pooling the two single-CS conditions to measure the effects of the previous CS type (compound versus singleton, dark and light pink, respectively) and current CS (filled and empty bars). Error bars represent SEM. 
316 tone or light alone. We observed a significant Pavlovian effect of CS type on these no-feedback

317 trials $\left[F(2,42)=4.78, p=0.014, \eta_{p}^{2}=0.19\right]$, with larger hand angles observed on compound CS trials

318 relative to the tone-alone or light-alone trials, conforming to the first prediction of the additivity

319 principle (Fig. 3B, 3C). Crucially, the additivity principle posits that there should be a negative

320 correlation between the associative strengths of competing CSs (Rescorla and Wagner, 1972).

321 That is, if a strong associative bond is formed between one CS and the US, this will come at the

322 expense of the associative strength accrued by any competing CSs (Eq. 2) given that there is a

323 capacity limit of associability $(V$ ). This prediction was strikingly confirmed in an analysis of the

324 hand angle changes on tone-alone and light-alone trials: Participants who were more sensitive to

325 the tone stimulus were less sensitive to the light stimulus, and vice versa (Fig. 3D, Pearson

326 correlation: $r=-0.72, p<0.001)$.

As in Experiment 1, since the probe phase of Experiment 2 consisted of different types of

CSs presented randomly across trials, the behavior for a given trial should reflect not only the CS on trial $n$ but also the motor state on trial $n-1$ (which is also influenced by the CS on that trial). As a further test of compound conditioning, we pooled the two single-CS conditions to measure the

331 effects of the previous and current CS type (singleton versus compound) on the observed 332 changes in hand angle. We again observed the Pavlovian effect $[F(1,21)=21.2, p<0.001$, $\left.333 \eta_{p}{ }^{2}=0.50\right]$ ]: There was a relative increase in hand angle for the compound CS (Mean \pm SE, $\left.3340.24^{\circ} \pm 0.06^{\circ}\right)$ and a relative decrease for the single CSs $\left(-0.18^{\circ} \pm 0.06^{\circ}\right)$ (Fig. 3E). The main effect 335 of the CS on trial $n-1$ was not significant $\left[F(1,21)=0.012, p=0.915, \eta_{p}{ }^{2}=0.00\right]$ nor was the trial $n-1$ $336 \times$ trial $n$ interaction $\left[F(1,21)=0.276, p=0.605, \eta_{p}^{2}=0.01\right]$, presumably due to the elimination of trial337 by-trial adaptation given the absence of visual feedback in the probe phase. 


\section{DISCUSSION}

341 Sensorimotor adaptation and eyeblink conditioning have provided foundational paradigms for the

342 study of error-based sensorimotor learning. They have yielded a rich empirical foundation in the

343 development of theoretical and neural models of learning and memory, particularly with respect

344 to cerebellar function (Albus, 1971; Ito, 1984; Marr, 1969; Wolpert et al., 1998). Adaptation has

345 typically been modeled from a control engineering perspective, centered on the idea that changes

346 in behavior are driven by sensory prediction errors arising when the experienced feedback

347 deviates from that predicted by a forward model operating on an efference copy of the motor

348 command (Krakauer et al., 2019; Shadmehr and Krakauer, 2008; Wolpert and Flanagan, 2001).

349 In contrast, eyeblink conditioning is treated as an associative learning process that can be driven

350 by arbitrary sensory cues.

Our goal in the present study was to take a first step towards establishing explicit links between these two frameworks. To that end, we asked if core phenomena identified in the study of classical conditioning are operative in sensorimotor adaptation. We showed that visuomotor

354 adaptation exhibited the hallmarks of both differential conditioning (Experiment 1) and compound

357 influence implicit feedforward motor adaptation and do so in a manner consistent with the 358 principles of associative learning rules.

The role of context in sensorimotor adaptation has been the subject of considerable

360 debate. A number of labs have employed sensory cues, asking if these are sufficient to negate 361 interference effects observed in response to interleaved opposing perturbations. The results from 362 this work have generally shown that arbitrary visual cues such as differently colored cursors are 363 ineffective, resulting in considerable interference between the perturbations (Gandolfo et al., 364 1996; Karniel and Mussa-Ivaldi, 2002; Howard et al., 2012, 2013; but see Krouchev and Kalaska, 
2003; Osu et al., 2004; Addou et al., 2011). In contrast, postural and movement-related variables, such as different lead-in and follow-through movements, have been shown to act as reliable contextual cues for overcoming interference effects (Howard et al., 2012, 2013, 2015; Sheahan et al., 2016). One interpretation of these results has focused on the difference between static and dynamic cues, with the idea that the latter are incorporated into the motor state, allowing for the separation of distinct internal models (Howard et al., 2012, 2013). cues. There are several key features of our design that differ from prior work with static cues.

373 First, drawing on the eyeblink literature (Schneiderman and Gormezano, 1964; Smith et al., 1969),

374 we imposed a strong temporal constraint on the interval between the CS (the contextual cue) and

375 the US by having the cues serve as imperative signals and enforcing rapid response times. This 376 strict temporal constraint was absent in prior studies; For example, in Howard et al. (Howard et 377 al., 2013), the color cue was presented 1,000 ms before the imperative. Second, the salience of 378 the arbitrary cues was arguably increased in the present study by having them serve as movement 379 imperatives.

More broadly, we propose that an associative learning framework may provide a useful 381 lens for understanding various aspects of sensorimotor adaptation. Consider a standard 382 adaptation study in which there are no contextual cues: Adaptation is observed when feedback is 383 perturbed while the participant reaches to a visual target. In such tasks, the target itself is not only 384 a salient stimulus that defines the task goal and movement plan, but its onset usually serves as 385 the imperative for movement initiation. Under these conditions, the target can be viewed as one 386 (highly effective) CS. Given that RTs in these tasks are typically below 500 ms (Kim et al., 2019, 387 2018; Avraham et al., 2021), such designs might establish a tight temporal link between target 388 appearance and movement, echoing (perhaps inadvertently) the CS-US temporal constraints 389 essential for eyeblink conditioning (Schneiderman and Gormezano, 1964). 
More precisely, we propose that it is the movement plan itself, rather than the target cue, that constitutes the primary CS in standard adaptation tasks. While the plan and target usually coincide, this is not always the case. For example, with a contingent visuomotor rotation, participants often deliberately aim away from the target, especially when the perturbation is large

394 (Hegele and Heuer, 2010; Taylor et al., 2014). Recognizing this "re-aimed" plan as a CS provides

395 a parsimonious account of a number of phenomena. First, generalization from adaptation is

396 centered around the direction of the movement plan but not the target (Day et al., 2016; McDougle

397 et al., 2017). This phenomenon echoes generalization effects seen in eyeblink conditioning,

398 where variation of the CS (e.g., tone frequency) leads to parametric changes in CR probability

399 (Siegel et al., 1968). Second, even in the absence of distinct contextual movements (e.g. follow-

400 through), the activation of different motor plans can serve as efficient contextual cues, negating

401 interference effects from opposing perturbations (Sheahan et al., 2016). Third, an emphasis on

402 the plan is in accord with general models of cerebellar-dependent adaptation, where the prediction

403 that constitutes the basis for sensory prediction error is computed using an efference copy of the 404 motor intention (Blakemore et al., 2001; Gao et al., 2016; Kawato and Gomi, 1992; Wolpert et al., 405 1998).

To formally relate the eyeblink and adaptation worlds, we implemented the Rescorla-

407 Wagner model, a classic associative learning model that has been widely employed in the 408 classical and operant conditioning literature (Rescorla and Wagner, 1972). The success of this 409 model to capture the general features of the current data sets should not be surprising since the 410 model was developed to account for phenomena such as differential conditioning and compound 411 conditioning. Thus, it was obvious to us that the Rescorla-Wagner model would provide better fits 412 than the standard state-space model, given that the latter cannot capture contextual effects. In 413 theory, the standard state-space model can be modified such that different sensory cues become 414 associated with different states (Heald et al., 2018), allowing for context-dependent learning (e.g., 
415 differential conditioning). However, we emphasize that to account for compound conditioning, and

416 in particular, the additivity principle, a state-space model would essentially have to become a 417 variant of a Rescorla-Wagner model.

Although the Rescorla-Wagner model provides a ready account of how arbitrary sensory

419 cues, visual targets, and even movement plans may provide a CS to drive adaptation, there are

420 other phenomena in the sensorimotor adaptation literature that would not be accounted for by the

421 model, at least in its simplest form. One such effect is spontaneous recovery, the re-manifestation

422 of a previously adapted state in the absence of error feedback. A variant of the state-space model,

423 one that allows for multiple states with different learning and forgetting rates, can capture

424 spontaneous recovery (Smith et al., 2006). Second is the relationship between learning rate and

425 environmental consistency; learning is faster in response to a consistent versus inconsistent

426 perturbation (Albert et al., 2021; Avraham et al., 2020; Gonzalez Castro et al., 2014; Herzfeld et

427 al., 2014; Hutter and Taylor, 2018). Here, the state-space model has been modified to allow the

428 learning rate to vary with experience (Herzfeld et al., 2014). In its simplest form, the Rescorla-

429 Wagner model cannot account for these effects. However, recent efforts to model associative

430 learning have focused on how this framework may require more sophisticated, holistic

431 computational approaches that complement simple associative mechanisms with inference

432 processes (Collins and Frank, 2013; Gershman, 2015). A similar approach has been incorporated

433 in a recent work looking at contextual effects in sensorimotor adaptation, and could successfully

434 capture the aforementioned effects (Heald et al., 2020).

While our results highlight principles that address context-dependent motor adaptation,

436 they do not speak to the error-correcting adaptation algorithm itself. Conventional models of this

437 algorithm focus on the role of forward models in predicting future sensory states and updating 438 motor commands to reduce sensory prediction errors, which are signed error signals (Wolpert 439 and Ghahramani, 2000; but see Hadjiosif et al., 2021). Associative learning does not provide a 
440 mechanism for the error correction process (e.g., a directional change in reaching movement

441 given rotated feedback, or a well-timed eyeblink response to an airpuff); rather, it describes the

442 association between a context and an error signal. Speculatively, motor adaptation perhaps

443 operates as a lookup table of a multitude of context-error associations, built up from a lifetime of

444 experience with subtle movement errors, with behavioral adjustments determined by a built-in,

445 direction-sensitive error correcting mechanism (Herzfeld et al., 2018). A model of this form could

446 bring sensorimotor adaptation closer to more classic models of cerebellar learning and plasticity

447 (Albus, 1971; Ito, 1984; Marr, 1969). More generally, exploring the computational links between

448 different sensorimotor learning tasks will be critical for understanding general principles of motor

449 learning and their neural mechanisms.

451 METHODS

\section{Participants}

453 Thirty-eight healthy volunteers (aged 18-31 years; 31 females) participated in either Experiment

$4541(\mathrm{~N}=16)$ or Experiment $2(\mathrm{~N}=22)$. All participants were right-handed, as self-reported and verified 455 with the Edinburgh Handedness Inventory. The protocol was approved by the Institutional Review 456 Board at the University of California, Berkeley.

458 Experimental setup and task

459 The participant sat at a custom-made table that housed a horizontally mounted LCD screen (53.2 $460 \mathrm{~cm}$ by $30 \mathrm{~cm}$, ASUS), positioned $27 \mathrm{~cm}$ above a digitizing tablet ( $49.3 \mathrm{~cm}$ by $32.7 \mathrm{~cm}$, Intuos $4 \mathrm{XL}$; 461 Wacom, Vancouver, WA). The participant held in their right hand a hockey paddle that contained 462 an embedded digitizing stylus. The monitor occluded direct vision of the hand, and the room lights 
were extinguished to minimize peripheral vision of the arm. Reaching movements were performed by sliding the paddle across the tablet. The sampling rate of the tablet was $200 \mathrm{~Hz}$.

At the beginning of each trial, a white circle $(0.5 \mathrm{~cm}$ diameter $)$ appeared at the center of 466 the black screen, indicating the start location (Fig. 1A). The participant moved the stylus to the 467 start location. Feedback of hand position (i.e., the stylus position) was indicated by a white cursor 468 (0.3 cm diameter), provided only when the hand was within $1 \mathrm{~cm}$ of the start location. A single blue target $(0.5 \mathrm{~cm}$ diameter) was positioned $8 \mathrm{~cm}$ from the start location. In most studies of

470 adaptation, the appearance of the target specifies both the movement goal (where to reach) and

471 serves as the imperative (when to reach). From a classical conditioning perspective, the target

472 should constitute a very salient CS given that its onset is temporally contingent with the US, the

473 visual feedback associated with the movement (see below). To eliminate this temporal 474 contingency, the target remained visible at the same location during the entire experiment. For 475 each participant, the target was placed at one of four locations, either $45^{\circ}, 135^{\circ}, 225^{\circ}$, and $315^{\circ}$, 476 and this location was counterbalanced across participants.

Movement initiation was cued by the presentation of the neutral (non-spatial) CS(s). We 478 used two different CSs, a tone and a light, both of which have no inherent association with the 479 US. The tone CS was a pure sine wave tone with a frequency of $440 \mathrm{~Hz}$. The light CS was a white 480 rectangular frame $[39.4 \mathrm{~cm} \times 26.2 \mathrm{~cm}]$ that spanned the perimeter of the visual workspace. The 481 large frame was selected to provide a salient visual stimulus, but one that would not be confused 482 with the target. The onset of the CS occurred following a pseudo-random and predetermined 483 delay after the hand was positioned at the start location. This was done to mitigate predictions 484 regarding the timing of the CS onset, and thus to enhance its salient role as an imperative. The 485 delay ranged between $800-1,200 \mathrm{~ms}$ (in steps of $100 \mathrm{~ms}$ ), and was drawn from a uniform 486 distribution. 

alone or together on a trial. The onset of the CS served as the imperative signal, with the participant instructed to rapidly reach directly towards the target, slicing through the target. The

490 CS was terminated when the hand reached $8 \mathrm{~cm}$, the radial distance to the target (Fig. 1A). To

491 minimize the delay between the onset of the CS and the US (the error clamp), the auditory 492 message "start faster" was played whenever a reaction time (RT) exceeded 400 ms. RT was 493 operationalized as the interval between CS onset and the time required for the radial distance of

494 the hand to exceed $1 \mathrm{~cm}$. Given our objective to test the link between feedforward adaptation and 495 classical conditioning, we sought to eliminate online feedback corrections. Participants were 496 instructed to make rapid movements and the auditory message "move faster" was played 497 whenever movement time exceeded $300 \mathrm{~ms}$. The end of the movement was operationalized as 498 the point where the radial distance of the hand reached $8 \mathrm{~cm}$.

For the unconditioned stimulus (US), we used task-irrelevant clamped feedback

500 (Morehead et al., 2017). With clamped feedback, the radial position of the visual cursor is matched

501 to the radial position of the hand. However, the angular position of the cursor is fixed. The 502 participant thus controlled the speed and radial distance of the cursor, but not its direction. When 503 designed to produce a prediction error and elicit implicit sensorimotor adaptation, the clamp 504 followed a path that deviated from the target by $15^{\circ}$, with the direction, i.e., clockwise $(\mathrm{CW})$ or 505 counterclockwise (CCW), counterbalanced across participants. We also included no-error trials 506 (Experiment 1 ) by presenting a clamped feedback that followed a path directly to the target $\left(0^{\circ}\right.$ 507 clamp; Fig. 1B). The nature of the clamp manipulation was described in detail to the participant, 508 and they were explicitly instructed strictly to ignore the feedback, aiming their reach directly toward 509 the target on every trial. These instructions were designed to emphasize that the participant did 510 not control the cursor position, and that they should always attempt to reach directly to the target. 511 The instructions of the task were reinforced by the presentation of short video animations to 
512 demonstrate how the CSs would serve as imperative signals, as well as to show the invariant

513 direction of the clamped feedback.

514 The experimental software was custom written in Matlab (The MathWorks, Natick, MA),

515 using the Psychtoolbox package (Brainard, 1997).

517 Experimental protocol

518 Both Experiments 1 and 2 included an acquisition phase and a probe phase. During the 519 acquisition phase, clamped feedback was presented on each trial, serving as the US. During the 520 probe phase, the clamped feedback was not presented. In both phases, the participants were 521 instructed to reach straight to the target as soon as the imperative CS appeared. Note that we 522 opted to not include baseline reaching blocks prior to the start of the acquisition phases to avoid 523 introducing any incidental associations between the baseline feedback and the target, movement 524 plan, and any other contextual variables. A break of approximately 1-minute was provided in the 525 middle of the experiment.

\section{Experiment 1: Differential conditioning}

527 Experiment $1(\mathrm{~N}=16)$ was designed to test differential conditioning in the context of a sensorimotor 528 adaptation task. The session consisted of 800 trials: 600 acquisition trials followed by 200 probe 529 trials (Fig. 1B). One of two CSs (tone or light) was presented on each trial, serving as the 530 imperative for the reaching response. During the acquisition phase, one CS was paired with a $15^{\circ}$ 531 clamped error feedback (CS+ condition) and the other CS was paired with a $0^{\circ}$ clamped feedback 532 (CS- condition). Each CS was presented on $50 \%$ of the trials, and the assignment of the tone and 533 light to the CS+ and CS- was counterbalanced across participants. During the probe phase, each 534 CS was presented alone on half of the trials, and there was no visual feedback. 
For both the acquisition and probe phases, the CS+ and CS- trials were interleaved in a

536 pseudo-random order that was unique to each participant. To ensure that the participant would

537 not be able to predict the CS type, the generated trial sequence for each participant was assessed

538 to verify that there was no significant lag-1 autocorrelation in the time series. At the end of the

539 experimental session, we assessed whether participants were aware of the contingency between

540 each CS and its associated feedback, asking, "Did you identify any pattern in the experiment?" in

541 a free-response survey.

\section{Experiment 2: Compound conditioning}

543 The adaptation task was modified in Experiment $2(\mathrm{~N}=22)$ to provide a test of compound

544 conditioning. The procedure was similar to that used in Experiment 1 with the following changes.

545 First, the session consisted of 600 acquisition trials and 300 probe trials (Fig. 3A). Second, a

546 compound CS, consisting of both the tone and light, served as the imperative throughout the

547 acquisition phase, and was always paired with a $15^{\circ}$ clamped feedback. Third, the probe phase

548 (no visual feedback) consisted of 100 trials for each of the compound CS, tone alone CS, and 549 light alone CS.

551 Data analysis

552 The recorded position of the digitizing stylus was analyzed using custom-written MATLAB scripts.

553 Our main analyses focused on the reach direction (hand angle) and the trial-by-trial changes in

554 hand angle ( $\Delta$ hand angle). Hand angle was defined by two imaginary lines, one from the start

555 position to the target and the other from the start position to the hand position at maximum

556 movement velocity.

Trials in which the hand angle was larger than $100^{\circ}$ off from the target, or in which the

558 trial-to-trial change in hand angle was larger than $25^{\circ}$, were considered outliers and not included 
559 in the analyses. These outliers constituted $0.03 \%$ and $0.16 \%$ of all trials in Experiments 1 and 2 ,

560 respectively. For the change in hand angle analysis, but not for the presentation of hand angle

561 time courses (Fig. S1), we excluded trials in which the reaction time exceeded $400 \mathrm{~ms}$ and/or

562 movement time exceeded 300 ms (Experiment 1: 11\% of acquisition trials, $4.3 \%$ of probe trials;

563 Experiment 2: 3.4\% of acquisition trials, $4.9 \%$ of probe trials).

For all analyses and to visualize the results, the sign of the hand angle was flipped for participants who experienced a CCW clamp, such that a positive hand angle is in the direction of expected adaptation (i.e., opposite the direction of the perturbed feedback). Moreover, the hand angle on the first acquisition trial was treated as the baseline reaching angle and subtracted from the hand angle on all subsequent trials. (We note that the results remain unchanged in terms of statistical comparisons if this baseline subtraction step is omitted.)

In Experiment 1, the primary analyses examined how the $\Delta$ hand angle was influenced by

571 the CS type (CS+ vs CS-), either in terms of the previous trial ( $n-1$, adaptation effect) or current 572 trial ( $n$, Pavlovian effect). For each participant and phase, we calculated the average $\Delta$ hand angle

573 for four types of trials: CS+ trials that follow CS+ trials, CS- after CS+, CS+ after CS-, and CS-

574 after CS-. For each phase, a two-way repeated-measures ANOVA was conducted with two within-

575 participant independent factors, the CS on trial $n-1$ and the CS on trial $n$, each with two levels,

576 CS+ and CS-. The $\Delta$ hand angle was the dependent variable in the two ANOVAs. To examine

577 the dynamics of the adaptation and Pavlovian effects (Fig. 2B), we binned the hand angle data

578 into epochs of 50 trials. Within each bin, we performed multiple regression analysis to test whether

579 trial-to-trial change in hand angle can be predicted from the previous trial CS, the current trial CS

580 and their interaction. Figure $2 B$ presents the mean \pm SEM regression $\beta$ weights of all simulated

581 time courses for the previous (adaptation effect) and current (Pavlovian effect) predictors. To

582 evaluate statistically the changes in $\beta$ weights for each type of effect across bins, we used a linear 
583 mixed model (R statistical package: ImerTest), with Type (adaptation and Pavlovian) and Bin as

584 fixed effects and participants as random effects.

585 To assess whether the Pavlovian effect is influenced by the awareness about the 586 contingency between each CS and its respective feedback, we divided the participants based on

587 their responses to the post experiment questionnaire (see Experimental protocol): 7 out of the 16

588 participants stated the correct contingency between the CS and the visual feedback and were

589 thus considered the "aware sub-group." The rest of the participants $(N=9)$ reported that they did

590 not identify any pattern related to the CS-US contingency and were considered the "unaware sub-

591 group." Independent two-sample $t$ tests were used to compare the Pavlovian effects between

592 these groups during the acquisition and probe phases of the experiment. For these tests we also

593 report Bayes factor $\mathrm{BF}_{10}$, the ratio of the likelihood of the alternative hypothesis $\left(\mathrm{H}_{1}\right)$ over the null

594 hypothesis $\left(H_{0}\right)$ (Kass and Raftery, 1995).

595 Feedback correction was operationalized as the difference between the hand angle

596 measured at the radial distance to the target and at $50 \mathrm{~ms}$ after movement initiation. We estimated

597 the mean and standard deviation of feedback correction across all acquisition trials in all of the

598 participants in Experiment 1. In addition, we calculated, for each participant, the mean feedback

599 correction for each of the CS+ and CS- trials, and used a paired-sample $t$ test to examine within-

600 participant changes in feedback correction between the two trial types.

In Experiment 2, the analysis focused on the probe phase in which there was no visual

602 feedback. We compared the $\Delta$ hand angle in response to the three CSs on trial $n$ (compound CS,

603 light alone, tone alone) regardless of the CS presented on trial $n-1$. We fit a one-way repeated-

604 measures ANOVA, with the $\Delta$ hand angle as the dependent variable, and the CS type as the 605 within-participant independent variable. 
The additivity principle of the Rescorla-Wagner model states that the association strengths

607 of each element of the compound CS will compete for associative strength with respect to the US

608 (Eq. 2). This principle was tested in our data by examining the correlation between the $\Delta$ hand 609 angle associated with the tone and light CSs in Experiment 2.

610 We report effect size using Cohen's $d$ for all $t$ tests and partial eta-squared $\left(\eta_{\mathrm{p}}{ }^{2}\right)$ for the

611 ANOVA. All of the measurements met the assumption of normality based on the Lilliefors test

612 (Lilliefors, 1967).

614 Model simulations

615 Trial-by-trial reach angles were simulated using the Rescorla-Wagner model as a representative

616 model for associative learning. In this model, the motor state is updated based on the associative

617 strength ( $V$ between the US (error signal) and all the CSs present on a given trial (Eqs. 1-2). The

618 extent of learning is determined by the maximum conditioning level $(\lambda)$, and the rate of behavioral

619 change are determined by the learning rate of the US $(\beta)$ and the salience of each presented CS

620 (a). To illustrate the predictions of the model in Figures 1E, 1F and 3C, we chose parameters that

621 result in qualitatively similar effects to the experimental results. For both Experiments 1 and 2, we

622 set $\lambda$ to 15 , similar to the observed implicit adaptation asymptotes these experiments, as well as

623 in other studies (Bond and Taylor, 2015; Morehead et al., 2017). The value of $\beta$ was set to 0.12

624 (Exp. 1) or 0.02 (Exp. 2), the salience parameter $(\alpha)$ for the movement plan CS to 0.99, and both

625 the tone CS and light CS to 0.002 (Exp. 1) or 0.1 (Exp. 2). These divergent salience values are

626 consistent with the assumption that most of the associative strength of the US would be absorbed

627 by the movement plan CS given its central relevance to the task of reaching to a target. The

628 remainder of potential associative strength to the US is thus available for the tone and light CSs. 
630 influences the predicted Pavlovian effects, we simulated behavior using various combinations of

631 the free parameters. We chose several combinations of values for $\beta$ and $\lambda$, and, for each

632 combination, a wide range of values for $\alpha_{\text {goal, }} \alpha_{\text {tone }}$ and $\alpha_{\text {light. }}$ (For simplicity, we constrained $\alpha_{\text {tone }}$

633 and $\alpha_{\text {light }}$ to the same value.) Figure S2 displays heatmaps resulting from these simulations, with

634 the color of each cell corresponding to the simulated difference in the trial-by-trial change in hand 635 angle between CS+ and CS- trials. In addition, we illustrated that a single-process state-space 636 model, a standard model of motor adaptation, cannot capture these Pavlovian effects. In this 637 model of motor adaptation, the motor state $(x)$ is updated according to the following learning rule 638 (Eq. 3):

(3) $\quad x^{[n]}=A \cdot x^{[n-1]}+B \cdot S P E^{[n-1]}$

640 where SPE is the sensory prediction error - the difference between the predicted and the actual 641 sensory feedback - experienced on trial $n-1, A$ is the retention factor, and $B$ is the learning rate.

642 For the simulation presented in Figure S2, the error was fixed to one of two values during the 643 acquisition phase, either $-15^{\circ}$ on CS + trials or $0^{\circ}$ on CS- trials, and in the probe phase, it was set 644 to $0^{\circ}$ on all trials.

While the two models share similar features and parameters (e.g., learning rate of an error 646 signal), the additional parameters in the Rescorla-Wagner model allow it to capture the effects of 647 differential and compound conditioning, should these processes be operative in our experiments. 648 For example, in the differential conditioning case, modifying the parameters of the Rescorla649 Wagner model could produce changes in the magnitude of both the within-trial "Pavlovian" effects 650 (i.e., the modulation of behavior in response to the current CS, or "trial $n$ " effects) and across-trial 651 adaptation effects (i.e., the modulation of behavior in response to the current state of learning, or 652 "trial $n$-1" effects). However, the Rescorla-Wagner model will always yield unique responses to 653 the CS+ and CS- given non-zero salience parameters. In contrast, no combination of the $A$ and 
$654 B$ parameters in the state-space model will produce differential responses to the tone and light

655 CSs.

656 To simulate the dynamics of the adaptation and Pavlovian effects in differential 657 conditioning (Fig. 2A, S4), and to compare it to the dynamics observed in the actual data, we 658 simulated the trial-by-trial change in hand angle during acquisition (600 trials) according to the 659 Rescorla-Wagner model (Eqs. 1 and 2) and the state-space model (Eq. 3), based on the 660 schedules of CS+ and CS- trials that were presented to the participants (16 simulated time 661 courses). For the Rescorla-Wagner model, we used the same parameters values that generated

662 the simulation results presented in Figures 1E and 1F. For the state-space model, we set the $A$ 663 parameter to 0.9 and $B$ to 0.12 . We then did the same trial-by-trial regression analysis described 664 above for the actual hand angle data (see Data analysis) with the exception that the dependent 665 variable was now the simulated change in hand angle.

Model fitting and comparison

668 We conducted a post-hoc model comparison analysis (Fig. S2). In this analysis, we fit the 669 Rescorla-Wagner model (Eqs. 1 and 2) and the standard state-space model (Eq. 3) to 670 participants' hand angle time course data of Experiment 1. The two models were fit by minimizing

671 the sum of squared residuals between the measured and modeled movement data, using the 672 MATLAB function fmincon. To avoid local minima, 200 randomized sets of initial parameter values 673 were used during fitting and the best fit of each model was selected for model comparison. Models 674 were compared using both the sum of squared residuals and the Akaike Information Criterion 675 approximated on the residuals (AIC; Akaike, 1974). All free parameters were bound at [0, 1], with 676 the exception of $\lambda$, which was bounded at $[-30,60]$. We did not perform model fitting for 677 Experiment 2 due to the fact that each trial was identical in the acquisition phase. As such, the 
678 salience parameters in the Rescorla-Wagner model for the goal, tone and light are unidentifiable,

679 and both models make indistinguishable behavioral predictions during acquisition.

680

681 ACKNOWLEDGEMENTS

682 We thank the Alkis Hadjiosif, John Krakauer, Adrian Haith and other members of the BLAM lab

683 (Johns Hopkins Medicine) for helpful discussions. We thank Marina Iranmanesh, Janet Hwang,

684 and Sarvenaz Pakzad for their assistance with data collection. RBI is funded by the NIH

685 (NS116883, NS105839 and DC077091). 


\section{REFERENCES}

687 Addou T, Krouchev N, Kalaska JF. 2011. Colored context cues can facilitate the ability to learn and to switch between multiple dynamical force fields. Journal of Neurophysiology 106:163-183. doi:10.1152/jn.00869.2010

Akaike H. 1974. A new look at the statistical model identification. IEEE Transactions on

Albert ST, Jang J, Sheahan HR, Teunissen L, Vandevoorde K, Herzfeld DJ, Shadmehr R. 2021. An implicit memory of errors limits human sensorimotor adaptation. Nature Human Behaviour 1-15. doi:10.1038/s41562-020-01036-x

Albus JS. 1971. A theory of cerebellar function. Mathematical Biosciences 10:25-61. doi:10.1016/0025-5564(71)90051-4

Avraham G, Keizman M, Shmuelof L. 2020. Environmental consistency modulation of error sensitivity during motor adaptation is explicitly controlled. Journal of Neurophysiology

Avraham G, Morehead JR, Kim HE, Ivry RB. 2021. Reexposure to a sensorimotor perturbation produces opposite effects on explicit and implicit learning processes. PLOS Biology 19:e3001147. doi:10.1371/journal.pbio.3001147

703 Blakemore S-J, Frith CD, Wolpert DM. 2001. The cerebellum is involved in predicting the sensory consequences of action. NeuroReport 12:1879-1884.

705 Bond KM, Taylor JA. 2015. Flexible explicit but rigid implicit learning in a visuomotor adaptation task. Journal of Neurophysiology 113:3836-3849. doi:10.1152/jn.00009.2015

707 Brainard DH. 1997. The Psychophysics Toolbox. Spatial Vision 10:433-436. 
Brudner SN, Kethidi N, Graeupner D, Ivry RB, Taylor JA. 2016. Delayed feedback during sensorimotor learning selectively disrupts adaptation but not strategy use. Journal of Neurophysiology 115:1499-1511. doi:10.1152/jn.00066.2015

Clark RE, Manns JR, Squire LR. 2002. Classical conditioning, awareness, and brain systems. Trends in Cognitive Sciences 6:524-531. doi:10.1016/S1364-6613(02)02041-7

Clark RE, Squire LR. 1998. Classical Conditioning and Brain Systems: The Role of Awareness. Science 280:77-81. doi:10.1126/science.280.5360.77

Collins AGE, Frank MJ. 2013. Cognitive control over learning: Creating, clustering, and generalizing task-set structure. Psychological Review 120:190-229.

Day KA, Roemmich RT, Taylor JA, Bastian AJ. 2016. Visuomotor Learning Generalizes Around the Intended Movement. eNeuro 3. doi:10.1523/ENEURO.0005-16.2016

Donchin O, Rabe K, Diedrichsen J, Lally N, Schoch B, Gizewski ER, Timmann D. 2011. 93:3843-3846. doi:10.1073/pnas.93.9.3843 FE, De Zeeuw CI. 2016. Excitatory Cerebellar Nucleocortical Circuit Provides Internal Amplification during Associative Conditioning. Neuron 89:645-657. doi:10.1016/j.neuron.2016.01.008

732 Garcia KS, Steele PM, Mauk MD. 1999. Cerebellar Cortex Lesions Prevent Acquisition of 733 Conditioned Eyelid Responses. J Neurosci 19:10940-10947.

734 doi:10.1523/JNEUROSCI.19-24-10940.1999 
Gershman SJ. 2015. A Unifying Probabilistic View of Associative Learning. PLoS Comput Biol 11. doi:10.1371/journal.pcbi.1004567

Gerwig M, Kolb FP, Timmann D. 2007. The involvement of the human cerebellum in eyeblink conditioning. Cerebellum 6:38. doi:10.1080/14734220701225904

Giurfa M. 2007. Behavioral and neural analysis of associative learning in the honeybee: a taste from the magic well. J Comp Physiol A 193:801-824. doi:10.1007/s00359-007-0235-9

Gonzalez Castro LN, Hadjiosif AM, Hemphill MA, Smith MA. 2014. Environmental Consistency Determines the Rate of Motor Adaptation. Current Biology 24:1050-1061.

Hadjiosif AM, Krakauer JW, Haith AM. 2021. Did we get sensorimotor adaptation wrong? Implicit adaptation as direct policy updating rather than forward-model-based learning. $J$

Heald JB, Ingram JN, Flanagan JR, Wolpert DM. 2018. Multiple motor memories are learned to control different points on a tool. Nature Human Behaviour 2:300-311. doi:10.1038/s41562-018-0324-5

Heald JB, Lengyel M, Wolpert DM. 2020. Contextual inference underlies the learning of sensorimotor repertoires. bioRxiv 2020.11.23.394320. doi:10.1101/2020.11.23.394320

752 Hegele M, Heuer H. 2010. Implicit and explicit components of dual adaptation to visuomotor rotations. Consciousness and Cognition 19:906-917. doi:10.1016/j.concog.2010.05.005

754 Herzfeld DJ, Kojima Y, Soetedjo R, Shadmehr R. 2018. Encoding of error and learning to correct that error by the Purkinje cells of the cerebellum. Nature Neuroscience 21:736-

757 Herzfeld DJ, Vaswani PA, Marko M, Shadmehr R. 2014. A memory of errors in sensorimotor learning. Science 1253138. doi:10.1126/science.1253138 
759

760

761

762

763

764

765

766

767

768

769

770

771

772

773

774

775

776

777

778

779

780

781

782

783

Howard IS, Ingram JN, Franklin DW, Wolpert DM. 2012. Gone in 0.6 Seconds: The Encoding of Motor Memories Depends on Recent Sensorimotor States. J Neurosci 32:12756-12768. doi:10.1523/JNEUROSCI.5909-11.2012

Howard IS, Wolpert DM, Franklin DW. 2015. The Value of the Follow-Through Derives from Motor Learning Depending on Future Actions. Current Biology 25:397-401. doi:10.1016/j.cub.2014.12.037

Howard IS, Wolpert DM, Franklin DW. 2013. The effect of contextual cues on the encoding of motor memories. Journal of Neurophysiology 109:2632-2644. doi:10.1152/jn.00773.2012

Hutter SA, Taylor JA. 2018. Relative sensitivity of explicit reaiming and implicit motor adaptation. Journal of Neurophysiology 120:2640-2648. doi:10.1152/jn.00283.2018

Ito M. 1984. The Cerebellum and Neural Control. Raven Press.

Izawa J, Criscimagna-Hemminger SE, Shadmehr R. 2012. Cerebellar Contributions to Reach Adaptation and Learning Sensory Consequences of Action. J Neurosci 32:4230-4239. doi:10.1523/JNEUROSCI.6353-11.2012

Karniel A, Mussa-Ivaldi FA. 2002. Does the motor control system use multiple models and context switching to cope with a variable environment? Exp Brain Res 143:520-524. doi:10.1007/s00221-002-1054-4

Kass RE, Raftery AE. 1995. Bayes Factors. Journal of the American Statistical Association 90:773-795. doi:10.1080/01621459.1995.10476572

Kawato M, Gomi H. 1992. A computational model of four regions of the cerebellum based on feedback-error learning. Biol Cybern 68:95-103. doi:10.1007/BF00201431

Kehoe EJ, Horne AJ, Horne PS, Macrae M. 1994. Summation and configuration between and within sensory modalities in classical conditioning of the rabbit. Animal Learning \& Behavior 22:19-26. doi:10.3758/BF03199952 
784 Kehoe EJ, Schreurs BG. 1986. Compound conditioning of the rabbit's nictitating membrane response: Test trial manipulations. Bull Psychon Soc 24:79-81. doi:10.3758/BF03330509

Kim HE, Avraham G, Ivry RB. 2020. The Psychology of Reaching: Action Selection, Movement Implementation, and Sensorimotor Learning. Annu Rev Psychol. doi:10.1146/annurevpsych-010419-051053

Kim HE, Morehead JR, Parvin DE, Moazzezi R, Ivry RB. 2018. Invariant errors reveal limitations

Kim HE, Parvin DE, Ivry RB. 2019. The influence of task outcome on implicit motor learning. eLife 8:e39882. doi:10.7554/eLife.39882

795 Kim JJ, Thompson RE. 1997. Cerebellar circuits and synaptic mechanisms involved in classical eyeblink conditioning. Trends in Neurosciences 20:177-181. doi:10.1016/S01662236(96)10081-3

Kitazawa S, Kohno T, Uka T. 1995. Effects of delayed visual information on the rate and amount of prism adaptation in the human. $J$ Neurosci 15:7644-7652. doi:10.1523/JNEUROSCI.15-11-07644.1995

Krakauer JW, Hadjiosif AM, Xu J, Wong AL, Haith AM. 2019. Motor LearningComprehensive Physiology. American Cancer Society. pp. 613-663. doi:10.1002/cphy.c170043

Krouchev NI, Kalaska JF. 2003. Context-Dependent Anticipation of Different Task Dynamics: Rapid Recall of Appropriate Motor Skills Using Visual Cues. Journal of Neurophysiology

806 Lilliefors HW. 1967. On the Kolmogorov-Smirnov Test for Normality with Mean and Variance 807 Unknown. Journal of the American Statistical Association 62:399-402.

808 doi:10.1080/01621459.1967.10482916 
Mackintosh NJ. 1976. Overshadowing and stimulus intensity. Animal Learning \& Behavior 4:186-192. doi:10.3758/BF03214033

Marr D. 1969. A theory of cerebellar cortex. The Journal of Physiology 202:437-470.

$$
\text { doi:10.1113/jphysiol.1969.sp008820 }
$$

813 Mazzoni P, Krakauer JW. 2006. An Implicit Plan Overrides an Explicit Strategy during Visuomotor Adaptation. J Neurosci 26:3642-3645. doi:10.1523/JNEUROSCI.5317-

McDougle SD, Bond KM, Taylor JA. 2017. Implications of plan-based generalization in sensorimotor adaptation. Journal of Neurophysiology 118:383-393. doi:10.1152/jn.00974.2016

McDougle SD, Bond KM, Taylor JA. 2015. Explicit and Implicit Processes Constitute the Fast and Slow Processes of Sensorimotor Learning. J Neurosci 35:9568-9579. doi:10.1523/JNEUROSCI.5061-14.2015

McDougle SD, Ivry RB, Taylor JA. 2016. Taking Aim at the Cognitive Side of Learning in Sensorimotor Adaptation Tasks. Trends in Cognitive Sciences 20:535-544. doi:10.1016/j.tics.2016.05.002 Adaptation Revealed by Task-irrelevant Clamped Feedback. Journal of Cognitive Neuroscience 29:1061-1074. doi:10.1162/jocn_a_01108

Osu R, Hirai S, Yoshioka T, Kawato M. 2004. Random presentation enables subjects to adapt to two opposing forces on the hand. Nature Neuroscience 7:111-112.

831 Parvin DE, McDougle SD, Taylor JA, Ivry RB. 2018. Credit assignment in a motor decision making task is influenced by agency and not sensorimotor prediction errors. $J$ Neurosci 3601-17. doi:10.1523/JNEUROSCI.3601-17.2018

Pavlov IP. 1927. Conditioned reflexes, translated by GV Anrep. London: Oxford. 
835 Poh E, Al-Fawakari N, Tam R, Taylor JA, McDougle SD. 2021. Generalization of motor learning in psychological space. bioRxiv 2021.02.09.430542. doi:10.1101/2021.02.09.430542

837 Popa LS, Streng ML, Hewitt AL, Ebner TJ. 2016. The Errors of Our Ways: Understanding Error Representations in Cerebellar-Dependent Motor Learning. Cerebellum 15:93-103.

840 Rasmussen A, Jirenhed D-A, Hesslow G. 2008. Simple and Complex Spike Firing Patterns in Purkinje Cells During Classical Conditioning. Cerebellum 7:563. doi:10.1007/s12311-

Rescorla R, Wagner A. 1972. A theory of Pavlovian conditioning: Variations in the effectiveness of reinforcement and nonreinforcement. Classical conditioning II: Current research and

Schlerf JE, Xu J, Klemfuss NM, Griffiths TL, Ivry RB. 2012. Individuals with cerebellar degeneration show similar adaptation deficits with large and small visuomotor errors. Journal of Neurophysiology 109:1164-1173. doi:10.1152/jn.00654.2011

Schneiderman N, Gormezano I. 1964. Conditioning of the nictitating membrane of the rabbit as adjustments to a visuomotor rotation. Neurobiology of Learning and Memory 140:124133. doi:10.1016/j.nlm.2017.02.015

Shadmehr R, Krakauer JW. 2008. A computational neuroanatomy for motor control. Exp Brain

857 Shadmehr R, Smith MA, Krakauer JW. 2010. Error Correction, Sensory Prediction, and 858 Adaptation in Motor Control. Annual Review of Neuroscience 33:89-108.

859 doi:10.1146/annurev-neuro-060909-153135 
Sheahan HR, Franklin DW, Wolpert DM. 2016. Motor Planning, Not Execution, Separates Motor Memories. Neuron 92:773-779. doi:10.1016/j.neuron.2016.10.017

Sheahan HR, Ingram JN, Žalalytė GM, Wolpert DM. 2018. Imagery of movements immediately following performance allows learning of motor skills that interfere. Scientific Reports 8:14330. doi:10.1038/s41598-018-32606-9

Shmuelof L, Huang VS, Haith AM, Delnicki RJ, Mazzoni P, Krakauer JW. 2012. Overcoming

Siegel S, Hearst E, George N. 1968. Generalization gradients obtained from individual subjects following classical conditioning. Journal of Experimental Psychology 78:171-174. doi:10.1037/h0026178

Smith MA, Ghazizadeh A, Shadmehr R. 2006. Interacting Adaptive Processes with Different Timescales Underlie Short-Term Motor Learning. PLOS Biology 4:e179.

Smith MC, Coleman SR, Gormezano I. 1969. Classical conditioning of the rabbit's nictitating membrane response at backward, simultaneous, and forward CS-US intervals. Journal

877 Taylor JA, Krakauer JW, Ivry RB. 2014. Explicit and Implicit Contributions to Learning in a Sensorimotor Adaptation Task. J Neurosci 34:3023-3032.

880 Tsay JS, Avraham G, Kim HE, Parvin DE, Wang Z, Ivry RB. 2020a. The effect of visual uncertainty on implicit motor adaptation. Journal of Neurophysiology 125:12-22.

883 Tsay JS, Kim HE, Parvin DE, Stover AR, Ivry RB. 2021a. Individual differences in proprioception predict the extent of implicit sensorimotor adaptation. Journal of Neurophysiology 125:1307-1321. doi:10.1152/jn.00585.2020 
Tsay JS, Lee AS, Ivry RB, Avraham G. 2021b. Moving outside the lab: The viability of conducting sensorimotor learning studies online. bioRxiv 2021.01.30.181370. doi:10.1101/2021.01.30.181370

Tsay JS, Parvin DE, Ivry RB. 2020b. Continuous reports of sensed hand position during sensorimotor adaptation. Journal of Neurophysiology 124:1122-1130. doi:10.1152/jn.00242.2020

Vandevoorde K, Orban de Xivry J-J. 2019. Internal model recalibration does not deteriorate with age while motor adaptation does. Neurobiology of Aging 80:138-153. doi:10.1016/j.neurobiolaging.2019.03.020

Weiss SJ. 1972. Stimulus compounding in free-operant and classical conditioning: A review and analysis. Psychological Bulletin 78:189-208. doi:10.1037/h0032956

Wolpert DM, Flanagan JR. 2001. Motor prediction. Current Biology 11:R729-R732. doi:10.1016/S0960-9822(01)00432-8

899 Wolpert DM, Ghahramani Z. 2000. Computational principles of movement neuroscience. Nature Neuroscience 3:1212-1217. doi:10.1038/81497

901 Wolpert DM, Ghahramani Z, Jordan MI. 1995. An internal model for sensorimotor integration. Science 269:1880-1882. doi:10.1126/science.7569931 Sciences 2:338-347. doi:10.1016/S1364-6613(98)01221-2 
FIGURE CAPTIONS

Figure 1. Experiment 1: Differential Conditioning.

911 (A) Reaching task. Participants reach from a start location (white circle) to a target (blue dot).

912 Online feedback is provided by a cursor (white dot). The target is displayed in a fixed location for

913 the entire experiment (location varied across participants), and the direction of the cursor

914 trajectory is fixed ("clamped") on all trials. After a random delay, a tone or a light (white frame) is

915 presented, serving as the movement imperative. The cue persists for the duration of the

916 movement. (B) Experimental protocol. During acquisition (white background), a $15^{\circ}$ clamp

917 (CW/CCW, counterbalanced across participants) was associated with CS+ (e.g., a tone) and a $0^{\circ}$

918 clamp with CS- (e.g., a light; counterbalancing the associations with the tone and light across

919 participants). During the probe phase (gray background), CS+ and CS- were presented with no

920 feedback. Throughout the entire experiment, CS+ and CS- trials were randomly interleaved. (C,

921 D) Experimental results $(\mathrm{N}=16)$ for trial-by-trial change $(\Delta)$ in hand angle during the acquisition

$922(C)$ and probe $(D)$ phases. Left panels present analysis results for an adaptation effect (main

923 effect of trial $n-1$, dark vs light blue) and a Pavlovian effect (main effect of the presented CS on

924 the current trial $n$, filled vs empty bars). The black outlined bar (right panel) presents the Pavlovian

925 effect, i.e., the subtraction of hand angle changes between CS+ and CS- trials. (E, F) Rescorla-

926 Wagner model simulation results during the acquisition $(E)$ and probe $(F)$ phases are consistent

927 with the experimental results. Error bars represent SEM. Dots represent individual participants.

929 Figure 2. Dynamics of adaptation and Pavlovian effects.

930 Time course of the mean weights (regression $\beta$ ) of the adaptation (trial $n-1$, grey) and Pavlovian

931 (trial $n$, blue) effects in Experiment 1 as predicted by the Rescorla-Wagner model (A) and derived 
932 from fits of the experimental results (B). Solid lines in $B$ represent least squares regression lines.

933 Shaded region represent SEM.

935 Figure 3. Experiment 2: Compound Conditioning.

936 (A) During acquisition, a tone and light were presented simultaneously (Compound CS), serving

937 as the imperative signal for the reaching movement. They were paired on all trials with a $15^{\circ}$

938 clamp. During the probe phase, no feedback was provided, and the CSs were presented either

939 together (Compound CS) or alone (Single CS; tone or light). (B) Experimental results ( $N=22)$ for

940 the $\Delta$ hand angle during the probe phase, showing a positive relative change for the compound

941 CS on the current trial $n$ (filled bar) and a negative relative change for each of its elements (empty

942 bars). (C) Predictions from the Rescorla-Wagner model for trial-by-trial $\Delta$ hand angle during the

943 probe phase. Note that the two elements were assumed to have equal weight in the simulation.

944 (D) Scatter plot showing the between-participant trade-off in terms of the associative strength of

945 the two CSs (the dotted black line represents the unity line). (E) The $\Delta$ hand angle after pooling

946 the two single-CS conditions to measure the effects of the previous CS type (compound versus

947 singleton, dark and light pink, respectively) and current CS (filled and empty bars). Error bars 948 represent SEM. 


\section{Figure 1}

A

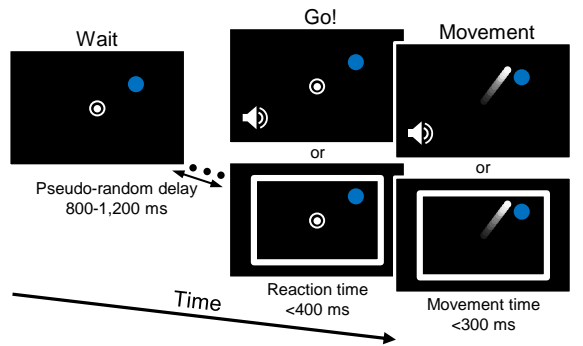

B

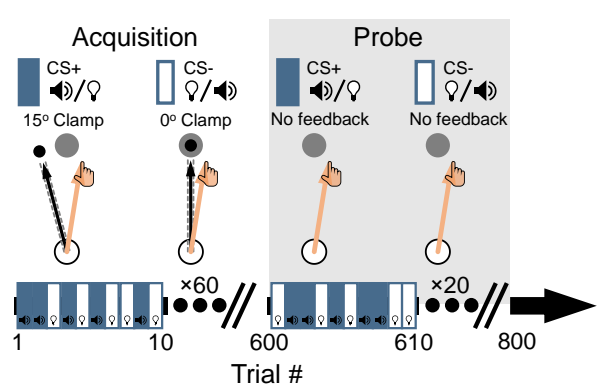

C

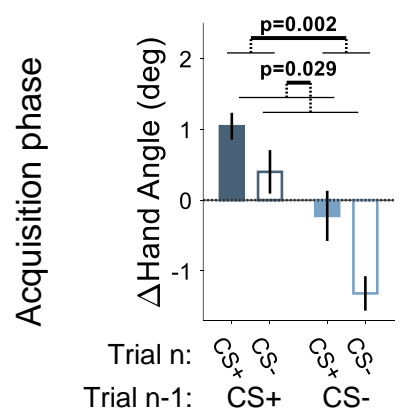

Trial n-1: CS+ CS-

D

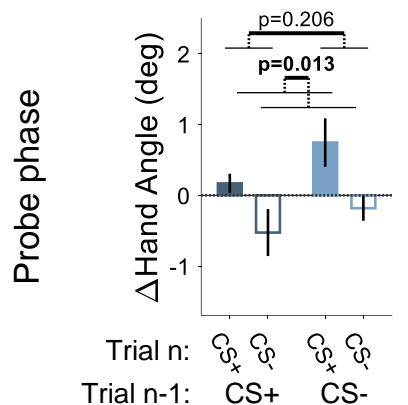

Pavlovian effect

CS+ - CS-

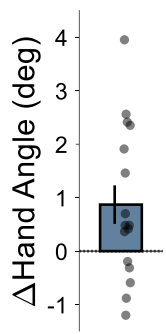

Pavlovian effect CS+ - CS-

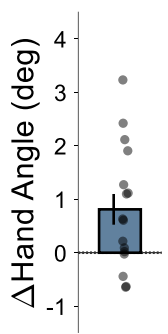

E

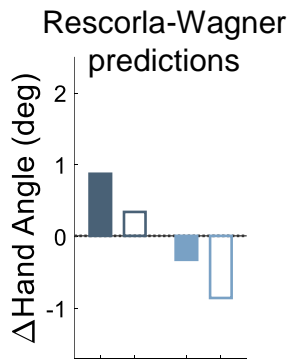

Trial n: $o_{x} o_{p} o_{x} o_{n}$

Trial n-1: CS+ CS-

F

Rescorla-Wagner

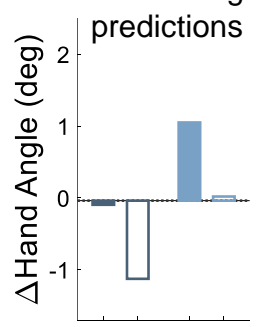

Trial n: of of of

Trial n-1: CS+ CS- 
Figure 2

A Rescorla-Wagner simulation
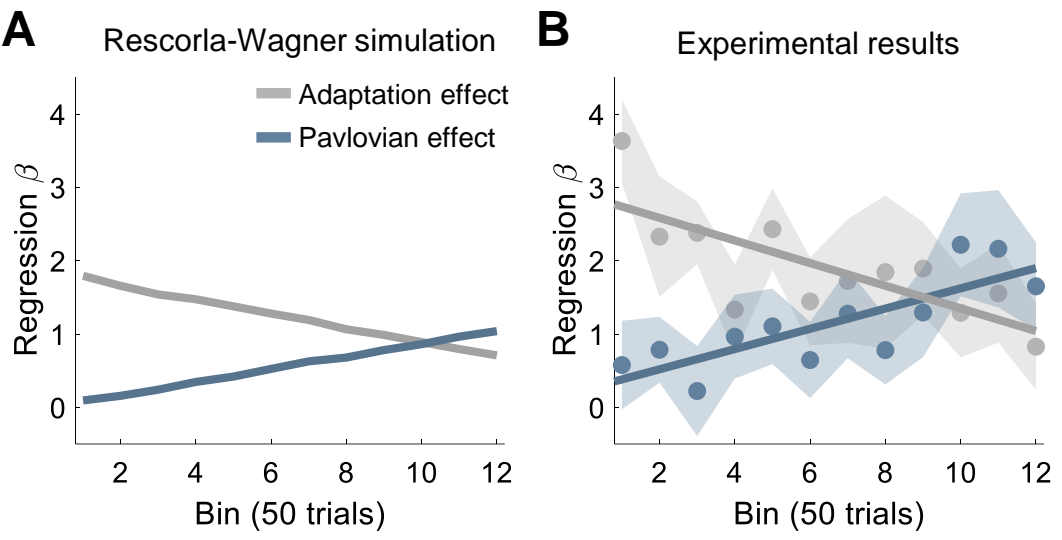
Figure 3

A

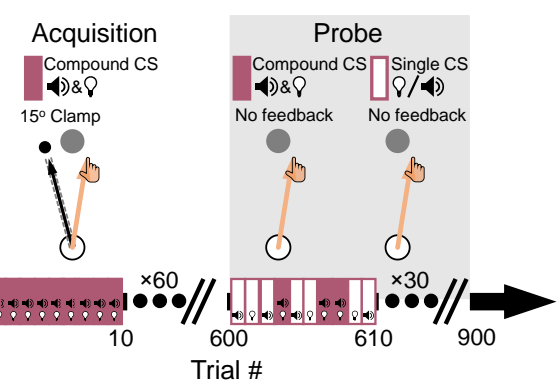

B

C

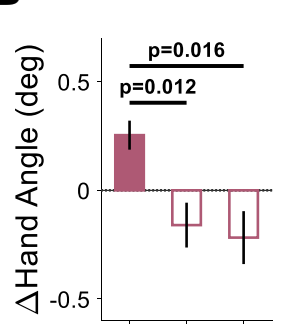

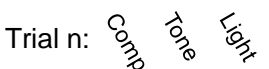

D
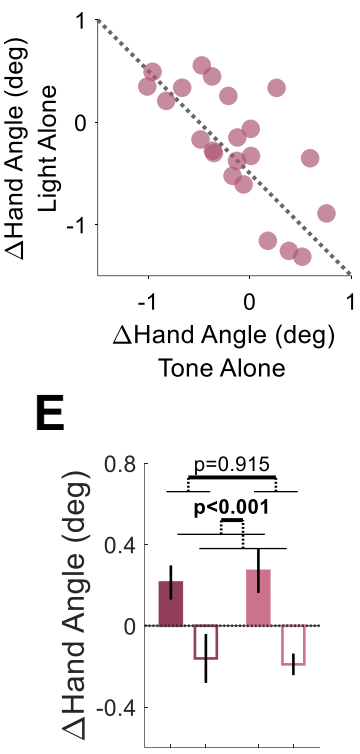

Trial $n$ :

Trial n-1: Comp Single 